\title{
The effects of cognitive training on gait speed and stride variability in old adults: findings from a pilot study
}

\author{
Jean-Paul Steinmetz $\cdot$ Carine Federspiel
}

Received: 25 March 2014/Accepted: 8 April 2014/Published online: 7 May 2014

(c) The Author(s) 2014. This article is published with open access at Springerlink.com

\begin{abstract}
Background The interrelationship between gait performance and higher-order cognitive functions has been established through a number of different investigations. In turn, enabling gait by improving cognition is a new and emerging field of research.

Aims Investigating if and to what extent a structured cognitive training program influences gait-related parameters in a sample of old and frail nursing home residents. Methods Twenty-one nursing home residents were quasirandomized to an intervention group following a 6-week structured cognitive training program or a control group. Gait was investigated during normal pace and under two dual-task conditions (simple and complex dual-task walking conditions), using the GAITRite ${ }^{\circledR}$ system at three predefined time points (pre-intervention, post-intervention, 3-month follow-up). Outcome measures were gait speed and stride variabilities.

Results Confirmation of the interrelationship between gait and cognition evidenced by decreased gait parameters during complex dual-task walking. Observation of clinical meaningful improvements in gait stability and gait speed after the training program under the complex dual-task situations, with only speed remaining stable over a period of 3 months. Discussion This study on the effects of cognitive training on gait is promising, with several results going in the expected direction. Our data corroborate previous findings
\end{abstract}

J.-P. Steinmetz $(\bowtie) \cdot C$. Federspiel

Department of Research and Development, ZithaSenior,

30 rue Ste Zithe, L-2763 Luxembourg, Luxembourg

e-mail: jean-paul.steinmetz@zitha.lu

C. Federspiel

Geriatric Rehabilitation Unit, ZithaKlinik, Luxembourg, Luxembourg and extend them to the group of frail old nursing home residents.

Conclusions The present pilot study's approach of improving gait under challenging walking situations by interventions designed to improve cognitions adds encouraging results to this emerging field of research, although restrictions in sample size and in the control group prevent us from drawing firm conclusions.

Keywords Cognitive training - Gait speed - Gait stability $\cdot$ Dual tasking

\section{Introduction}

In recent years, an increasing number of research studies focusing on the interplay between higher-order cognitive functions and gait in older adults suggest a causal link between cognitive dysfunctions, gait disorders and falls [15]. Age-associated degradation in motor functions is thought to be compensated by higher-order executive functions and attention. However and dramatically, when compensating for age-related motor declines older adults are facing a severe impasse as they have to rely on a deteriorating cognitive system [1].

Declines of gait and balance under dual-task (DT) situations have been observed in several studies, suggesting that walking is a complex motor task relying on high-level cognitive functions (for a review, see [6]). This view has been amplified by findings of a recent study, with participants experiencing alterations in almost all investigated gait measures (i.e., reduced velocity, increased variability) while walking and performing a second task, compared to a single walking condition [7]. In old adults, implications of higher cognitive function on gait seem, however, not to be 
restricted to a priori complex walking conditions. Data from Hausdorff and colleagues suggest that executive functions play a crucial role during the performance of normal and routine walking activities among older adults [8]. This leads to the conclusion that deficits in higher cognitive functions may result in an increased fall risk and hence, a compromised quality of life as was recently corroborated by research findings demonstrating that executive functions are associated with future falls for periods ranging up to 66 months [3, 4]. In sum, it is becoming largely accepted that gait is associated with higher-order cognitive functions in older adults [6, 8,9]. Given this, a relatively new approach investigates if benefits of cognitive training programs can potentially transfer to untrained functional domains [10], such as gait. A study by Verghese [11] reports positive transfer effects from trained cognitive functions to untrained gait-related parameters. The authors found increased gait velocities compared to baseline measures in their intervention group (IG) following an 8 -week training program (Cohen's $d=0.44$ ). No improvements were observed in the control group (CG) $(d=0.06)$. In another noteworthy study [12], positive effects of cognitive training on (instrumental) activities of daily living after a ten-year follow-up are reported. Hence and in sum, cognitive intervention programs on gait in older adults produced first results suggesting that cognitive interventions may enable old adults to successfully allocate their cognitive resources among two competing activities [3], thus improving their gait qualities. In the context of this emerging field, the present study therefore investigates if a previously validated cognitive intervention program [13] significantly improves gait parameters in frail old adults.

\section{Methods}

Participants and design

A total number of $N=355$ nursing home residents were screened for eligibility. Eligibility criteria were a Mini Mental State Examination score $\geq 23$, ability to walk without an ambulatory aid for more than $10 \mathrm{~m}$ and no substantial visual or hearing impairments unless corrected. Sixty-six individuals were identified meeting the inclusion criteria from which 42 provided informed consent. Our sample was quasi-randomized to two groups: one IG and one CG. Please refer to Fig. 1 for details on the flow of participants. Given the present applied research context and the pilot nature of the study, participants not willing to be attributed to the IG were asked to participate as CG participants in the study. We were unable to exclude participants demonstrating health concerns other than reduced cognitive functions. Participants were therefore screened for their frailty status (see Table 1 for a detailed overview). Gait parameter assessments were conducted in both study groups at three predefined moments: pre-intervention, postintervention, and 3 months after the completion of the intervention by the IG (follow-up). Of the initial sample size of 42 participants in the pre-intervention assessment, drop-outs reduced the number of participants in both groups to $N=21$ (12 IG and 9 CG; see Fig. 1). Only participants completing the three assessments (pre-intervention, post-intervention, follow-up) are included in the present analyses. The study was approved by the National Research Ethics Committee and written consent was obtained from all participants.

\section{Gait analysis}

Gait analyses were performed according to the published European guidelines [14] using a 518-cm long GAITRite ${ }^{\circledR}$ walkway (CIR Systems Inc., Clifton, NJ, USA). In total, three gait conditions were assessed, (a) normal walking, with participants instructed to walk at their habitual speed (single walking condition), (b) DT walking while counting out loud in an ascending order starting by 1 (simple DT condition), and (c) DT walking while counting backwards by twos out loud starting from a predefined number (complex DT condition). Under DT conditions, no explicit instructions on prioritization were given. Participants were instructed to perform two walks per condition, with the goal of increasing the number of steps to be analyzed and thus the precision of the measure. Gait parameters were collected without a walking aid [15], whereas participants were free to sit down and rest in between walks at their own convenience. The selection of gait parameters was limited to parameters that have previously been associated with gait dysfunctions and falls among old adults [7, 16]. Stride-to-stride variabilities are measured by the coefficient of variation $[\mathrm{CoV}=($ standard deviation/mean $) \times 100]$. For the present study, three gait parameters were retained for further analysis: velocity $(\mathrm{cm} / \mathrm{s}), \mathrm{CoV}$ of stride time (in $\%$ ), $\mathrm{CoV}$ of stride length (in \%).

\section{Cognitive training program}

The cognitive training program [13] is composed of 12 training sessions to be administered in a period of 6 weeks ( 2 sessions per week). Sessions last $90 \mathrm{~min}$ each and are thoroughly structured and standardized. Training sessions were administered and supervised by experienced trainers. The participants of the IG were split up into smaller groups of three to five people; the rate of attendance of the IG participants over the 12 training sessions was $86.7 \%$. Each session was composed of five different types of content, 


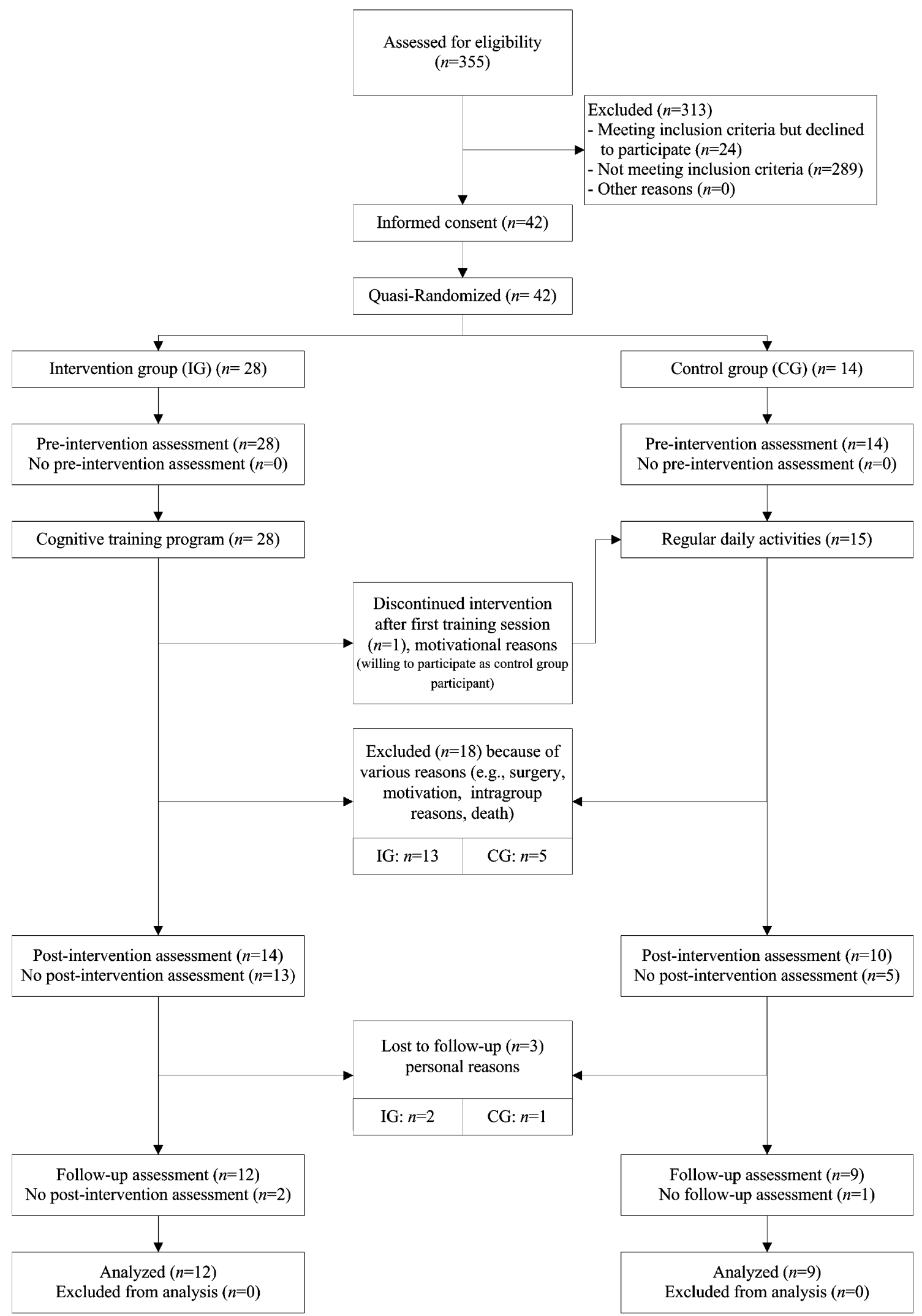

Fig. 1 Diagram to show the flow of study participants 
Table 1 Baseline characteristics

\begin{tabular}{|c|c|c|c|}
\hline & $\begin{array}{l}\text { Intervention } \\
(n=12)\end{array}$ & $\begin{array}{l}\text { Control } \\
(n=9)\end{array}$ & $\begin{array}{l}p \text { value } \\
\text { (univariate) }\end{array}$ \\
\hline Age, years & $83.8 \pm 6.5$ & $85.7 \pm 5.4$ & 0.50 \\
\hline Body mass index & $26.4 \pm 5.5$ & $25.5 \pm 5.5$ & 0.75 \\
\hline MMSE & $27.1 \pm 1.8$ & $27.6 \pm 2.0$ & 0.58 \\
\hline Barthel & $88.3 \pm 13.7$ & $95.6 \pm 5.3$ & 0.15 \\
\hline GDS-4 & $0.83 \pm 0.72$ & $0.44 \pm 0.73$ & 0.24 \\
\hline CIRS-G severity index & $1.8 \pm 0.45$ & $2.0 \pm 0.36$ & 0.46 \\
\hline Get Up and Go Test, s & $22.0 \pm 9.4$ & $19.3 \pm 5.7$ & 0.46 \\
\hline $\begin{array}{l}\text { Grip strength maximal, } \\
\mathrm{kg}\end{array}$ & $16.1 \pm 4.1$ & $19.8 \pm 7.7$ & 0.16 \\
\hline Falls in the last 6 months & $0.82 \pm 1.6$ & $1.1 \pm 1.6$ & 0.69 \\
\hline $\begin{array}{l}\text { Length of stay in facility, } \\
\text { months }\end{array}$ & $9.3 \pm 9.4$ & $51.3 \pm 97.5$ & 0.15 \\
\hline \multicolumn{4}{|l|}{ Participants, $n(\%)$} \\
\hline Females & $10(83.3)$ & $7(77.8)$ & 0.59 \\
\hline \multicolumn{4}{|l|}{ Education } \\
\hline$<13$ years & $11(91.7)$ & $8(88.9)$ & 0.69 \\
\hline$\geq 13$ years & $1(8.3)$ & $1(11.1)$ & \\
\hline $\begin{array}{l}\text { Taking more than } 6 \\
\text { drugs/day }\end{array}$ & $11(91.7)$ & $8(88.9)$ & 0.69 \\
\hline $\begin{array}{l}\text { With cardio-vascular } \\
\text { drugs }\end{array}$ & $7(58.3)$ & $5(55.6)$ & 0.62 \\
\hline $\begin{array}{l}\text { With psychoactive } \\
\text { drugs }\end{array}$ & $10(83.3)$ & $8(88.9)$ & 0.61 \\
\hline $\begin{array}{l}\text { Using a walking aid in } \\
\text { everyday life }\end{array}$ & $9(75.0)$ & $6(66.7)$ & 0.52 \\
\hline With fear of falling & $6(50.0)$ & $3(33.3)$ & 0.38 \\
\hline
\end{tabular}

All values are mean \pm SD unless otherwise stated. GDS-4, Geriatric Depression Scale, 4 item version [17]; CIRS-G severity index, Cumulative Illness Rating Scale for Geriatrics [18]; severity index $=($ total CIRS-G score/total number of categories endorsed $)$

(a) theoretical background on the topic of the day (i.e., meta-cognitive contents such as "how does my memory work"), (b) group exercises, (c) stimulating cognitive games, (d) individual exercises, and (e) home assignments. The cognitive abilities that were specifically trained via the training program are attentional capacities, working memory, the ability to plan, verbal fluency, learning and memory. The training program's effectiveness in mild cognitive impairment was recently discussed [19].

\section{Statistical analyses}

Statistical analyses are limited to a total of 21 participants ( $n=12$ in the IG and $n=9$ in the CG). Prior to analyzing the data, outlier detection was conducted using the boxplot outlier labeling rule [20]. Distribution assumptions of the data were verified by examining distribution histograms and values of skewness, kurtosis and by using the Kolmogorov-Smirnov test. To investigate the influence of the two DT conditions on gait speed, $\mathrm{CoV}$ stride time and $\mathrm{CoV}$ stride length, repeated measures MANOVA was performed for the single walking, the simple DT, and the complex DT conditions, which can be understood as within-subject factors for the pre-intervention assessment only. In case of a violation of sphericity, Greenhouse-Geisser corrected values are reported. Univariate pairwise comparisons using Bonferroni corrected dependent $t$ tests are used to uncover possible differences between the three walking conditions.

Mean differences between walking conditions are considered significant when $p$ value is $<0.05$. The effects of the intervention on the IG were investigated by computing repeated measures MANCOVAs with the three walking conditions as within-subject factors for each of the three time points (pre-intervention, post-intervention, followup). The between-subjects factor was the group variable (IG or CG), with gait parameters as dependent variables. The Barthel score, maximal handgrip strength and length of stay in facility were introduced in the model as possible confounders because the effect sizes between IG and CG for all three scores were found to be substantial. Cohen's $d s$ turned around 0.70 for the Barthel score and maximal handgrip strength and around 4.6 for length of stay.

To quantify the participants' ability to execute two tasks concurrently and to ease the interpretation of possible intervention effects, we computed DT costs for each subject according to the formula DTC $=((\mathrm{DT}$ walking normal walking/normal walking) $\times 100$ ). Hence, two DTC scores were computed, (a) the simple DTC score representing DT costs between the normal walking condition (single task) and the simple DT condition, and (b) the complex DTC score representing DT costs between the normal walking condition (single task) and the complex DT condition. Given the restricted sample size of the present study, statistical power of our analyses of (co)variances is limited. We therefore report Kazis effect sizes $\delta$ to quantify the amount of meaningful change in our gait parameters [21, 22]. Guidelines for interpreting an effect size are 0.2 for small, 0.5 for moderate, and 0.8 for large changes [23].

\section{Results}

Dual-task and gait

We find a significant main effect of the within-subjects factor walking condition on gait speed $(F(1.3$, $26.0)=12.00, p=0.001$ ), with participants walking significantly slower in the complex DT condition $(51.1 \mathrm{~cm} / \mathrm{s})$ compared to the single walking condition $(59.9 \mathrm{~cm} / \mathrm{s})$. A significant effect of walking condition on the coefficient of variation of stride time $(F(1.3,26.8)=6.32, p=0.01)$ is 
Table 2 Walking performances during the Three Walking Conditions with Pairwise Comparisons

\begin{tabular}{|c|c|c|c|c|c|c|c|c|}
\hline & & \multirow{2}{*}{$\begin{array}{l}\text { Single } \\
\text { walking }\end{array}$} & \multirow{2}{*}{$\begin{array}{l}\text { Simple } \\
\text { DT }\end{array}$} & \multirow{2}{*}{$\begin{array}{l}\text { Complex } \\
\text { DT }\end{array}$} & \multicolumn{2}{|c|}{ Single walking vs. simple DT } & \multicolumn{2}{|c|}{ Single walking vs. complex DT } \\
\hline & & & & & $\begin{array}{l}\text { Mean } \\
\text { difference } \\
(p \text { value })\end{array}$ & $\begin{array}{l}95 \% \mathrm{CI} \\
\text { of mean } \\
\text { difference }\end{array}$ & $\begin{array}{l}\text { Mean } \\
\text { difference } \\
(p \text { value })\end{array}$ & $\begin{array}{l}95 \% \mathrm{CI} \\
\text { of mean } \\
\text { difference }\end{array}$ \\
\hline Gait speed $(\mathrm{cm} / \mathrm{s})$ & $\mathrm{M}(\mathrm{SD})$ & $59.9(16.0)$ & $58.8(16.8)$ & $51.1(17.3)$ & $1.1(0.23)$ & $-1.9 ; 4.1$ & $8.9(0.003 *)$ & $3.5 ; 14.2$ \\
\hline $\mathrm{CoV}$ stride length (\%) & $\mathrm{M}(\mathrm{SD})$ & $6.9(4.7)$ & $6.5(3.2)$ & $8.6(4.2)$ & $0.4(0.28)$ & $-1.1 ; 1.9$ & $-1.7(0.10 \ddagger)$ & $-3.7 ; 0.4$ \\
\hline $\mathrm{CoV}$ stride time $(\%)$ & $\mathrm{M}(\mathrm{SD})$ & $4.9(3.0)$ & $5.0(2.4)$ & $7.9(4.7)$ & $-0.2(0.39)$ & $-1.2 ; 0.9$ & $-3.0(0.02 \dagger)$ & $-5.3 ;-0.6$ \\
\hline
\end{tabular}

$p$ values based on dependent $t$ test statistics, one-tailed

* Significant at the 0.01 significance level

$\dagger$ Significant at the 0.05 significance level

\$ Tentatively significant at the 0.10 significance level

furthermore observed, demonstrating that participants have a reduced gait stability under the complex DT condition (7.9\%) compared to the single walking condition (4.9\%). No significant differences in gait speed and stride time variabilities were observed between the single walking condition and the simple DT condition (see Table 2).

Cognitive training and gait

Analyses of covariance reveal no significant interaction effects on mean values between group and time of measurement on the three investigated gait parameters, gait speed, $\mathrm{CoV}$ stride time, and $\mathrm{CoV}$ stride length (ps $>0.18$; see Table 3).

Concerning DTC in gait speed, we observe encouraging results in the expected direction with small clinically meaningful effects in the IG between pre-intervention and post-intervention assessments $(\delta=\mid 0.261)$, suggesting a less dramatic reduction of gait speed under the complex DT situation after the intervention compared to prior the intervention. This effect persists somewhat up until 3 months after the end of the intervention, with $\delta=|0.16|$ between pre-intervention and follow-up assessments (Fig. 2a). Computed interactions between group and time of measurement for the single walking condition compared to the simple DT condition $(F(2,32)=1.38, p=0.27)$ and for the single walking condition compared to the complex DT condition $(F(2,32)=1.60, p=0.22)$ were non-significant. In the CG we observe an increasing DTC during the course of the study (total study period of approximately 18 weeks).

Concerning DTC in CoV stride time, no significant interaction effects between group and time of measurement were found ( $\mathrm{ps}>0.25$ ). Interestingly, one finding in the expected direction was observed, with our IG experiencing less complex DTC in stride time variability after the intervention $(93.0 \%)$ compared to the pre-intervention assessment (1.67 factor increase; see Fig. 2b). This change represents a small to medium-sized clinically meaningful effect $(\delta=0.45)$, which is, however, unstable in time as stride time $\mathrm{CoVs}$ are again largely increased during the follow-up assessment (2.10 factor increase). In the CG, DTC increases from $9.3 \%$ at the pre-intervention assessment up to $63.9 \%$ during the follow-up assessment.

Concerning DTC in CoV stride length, no significant interaction effects between group and time of measurement were found for simple DTC $(F(2,32)=2.52, p=0.10)$ and for complex DTC $(F(2,32)=2.34, \quad p=0.11)$. Although both interactions are non-significant, participants in the IG demonstrated important changes in their stride length variability at the post-intervention (16.7 and $25.6 \%$, respectively) assessment compared to the pre-intervention assessment (29.3 and $93.9 \%$, respectively), representing a small-sized clinically meaningful change for the simple DTC interaction $(\delta=0.25)$ and a medium- to large-sized change for the complex DTC interaction $(\delta=0.68$; see Fig. 2c). Simple DTC is observed to be further reduced during the follow-up assessment in the IG (effect size between pre-intervention and follow-up assessments, $\delta=0.54$ ), contrary to the magnitude of the complex DTC. This suggests a limited stability over time of the observed complex DTC effect, with effect sizes between pre-intervention and follow-up assessments of $\delta=0.24$. Again, the CG differs from the IG at baseline with DTCs increasing dramatically over the entire study period (from $5.0 \%$ at baseline up to a 1.17 factor increase at follow-up).

\section{Discussion}

We found evidence for the gait-cognition association, with participants reducing their gait speed and increasing their stride time variability (reduced gait stability) significantly under the complex walking situation compared to the single walking situation. Concerning the effects of cognitive training on gait parameters, clinically meaningful 
Table 3 Mean values, standard deviations, and $95 \%$ confidence intervals of gait speed, and coefficient of variations of stride time and stride length during single walking, simple dual-task (DT) walking, and complex DT walking conditions at pre-intervention, post-intervention, and follow-up assessments for both groups (IG, CG)

\begin{tabular}{|c|c|c|c|c|c|c|c|}
\hline \multirow[t]{2}{*}{ Gait speed (in $\mathrm{cm} / \mathrm{s}$ ) } & & \multicolumn{2}{|l|}{ Pre-intervention } & \multicolumn{2}{|c|}{ Post-intervention } & \multicolumn{2}{|l|}{ Follow-up } \\
\hline & & Mean \pm SD & $95 \%$ CI [LL; UL] & Mean $\pm \mathrm{SD}$ & $95 \%$ CI [LL; UL] & Mean \pm SD & $95 \%$ CI [LL; UL] \\
\hline \multirow[t]{2}{*}{ Single walking } & IG & $66.1 \pm 14.6$ & {$[57.1 ; 75.0]$} & $66.3 \pm 16.6$ & {$[56.1 ; 76.5]$} & $63.5 \pm 14.8$ & {$[54.4 ; 72.6]$} \\
\hline & CG & $51.7 \pm 15.0$ & {$[41.2 ; 62.3]$} & $61.1 \pm 17.0$ & {$[49.0 ; 73.1]$} & $60.7 \pm 15.2$ & {$[50.0 ; 71.5]$} \\
\hline \multirow[t]{2}{*}{ Simple DT walking } & $\mathrm{IG}$ & $63.0 \pm 16.7$ & {$[52.7 ; 73.2]$} & $62.3 \pm 15.0$ & {$[53.2 ; 71.5]$} & $60.5 \pm 17.5$ & {$[49.8 ; 71.2]$} \\
\hline & CG & $53.2 \pm 17.1$ & {$[41.1 ; 65.3]$} & $56.5 \pm 15.3$ & {$[45.7 ; 67.3]$} & $56.5 \pm 17.9$ & {$[43.9 ; 69.2]$} \\
\hline \multirow[t]{2}{*}{ Complex DT walking } & IG & $54.7 \pm 19.4$ & {$[42.9 ; 66.6]$} & $58.3 \pm 17.0$ & {$[47.9 ; 68.8]$} & $53.9 \pm 17.9$ & {$[42.9 ; 64.8]$} \\
\hline & CG & $46.2 \pm 19.8$ & {$[32.2 ; 60.2]$} & $49.8 \pm 17.5$ & {$[37.4 ; 62.1]$} & $49.1 \pm 18.3$ & {$[36.2 ; 62.1]$} \\
\hline \multirow{2}{*}{\multicolumn{2}{|c|}{ CoV stride time (in \%) }} & \multicolumn{2}{|c|}{ Pre-intervention } & \multicolumn{2}{|c|}{ Post-intervention } & \multicolumn{2}{|l|}{ Follow-up } \\
\hline & & Mean $\pm \mathrm{SD}$ & 95 \% CI [LL; UL] & Mean $\pm \mathrm{SD}$ & 95 \% CI [LL; UL] & Mean \pm SD & $95 \%$ CI [LL; UL] \\
\hline \multirow[t]{2}{*}{ Single walking } & IG & $3.8 \pm 2.1$ & {$[2.5 ; 5.1]$} & $3.6 \pm 1.4$ & {$[2.7 ; 4.4]$} & $4.4 \pm 2.0$ & {$[3.2 ; 5.7]$} \\
\hline & CG & $6.4 \pm 2.2$ & {$[4.9 ; 7.9]$} & $4.1 \pm 1.5$ & {$[3.1 ; 5.2]$} & $5.2 \pm 2.1$ & {$[3.8 ; 6.7]$} \\
\hline \multirow[t]{2}{*}{ Simple DT walking } & IG & $3.9 \pm 2.0$ & {$[2.7 ; 5.1]$} & $5.4 \pm 3.1$ & {$[3.5 ; 7.4]$} & $5.5 \pm 3.1$ & {$[3.6 ; 7.4]$} \\
\hline & CG & $6.6 \pm 2.0$ & {$[5.2 ; 8.1]$} & $6.0 \pm 3.2$ & {$[3.7 ; 8.2]$} & $6.3 \pm 3.2$ & {$[4.1 ; 8.6]$} \\
\hline \multirow[t]{2}{*}{ Complex DT walking } & IG & $8.6 \pm 5.6$ & {$\left[\begin{array}{ll}5.2 & 12.0\end{array}\right]$} & $6.5 \pm 3.5$ & {$[4.4 ; 8.6]$} & $11.1 \pm 7.0$ & {$[6.8 ; 15.4]$} \\
\hline & $\mathrm{CG}$ & $6.9 \pm 5.7$ & {$[2.9 ; 11.0]$} & $6.2 \pm 3.5$ & {$[3.7 ; 8.7]$} & $8.2 \pm 7.1$ & {$[3.2 ; 13.3]$} \\
\hline \multirow{2}{*}{\multicolumn{2}{|c|}{ CoV stride length (in \%) }} & \multicolumn{2}{|c|}{ Pre-intervention } & \multicolumn{2}{|c|}{ Post-intervention } & \multicolumn{2}{|l|}{ Follow-up } \\
\hline & & Mean \pm SD & $95 \%$ CI [LL; UL] & Mean \pm SD & $95 \%$ CI [LL; UL] & Mean \pm SD & $95 \%$ CI [LL; UL] \\
\hline \multirow[t]{2}{*}{ Single walking } & IG & $4.8 \pm 3.7$ & {$[2.5 ; 7.1]$} & $5.5 \pm 3.0$ & {$[3.6 ; 7.3]$} & $5.4 \pm 2.2$ & {$[4.0 ; 6.7]$} \\
\hline & CG & G $\quad 9.8 \pm 3.9$ & {$[7.1 ; 12.5]$} & $5.9 \pm 3.1$ & {$[3.7 ; 8.1]$} & $5.4 \pm 2.3$ & {$[3.8 ; 7.0]$} \\
\hline \multirow[t]{2}{*}{ Simple DT walking } & IG & $5.9 \pm 2.3$ & {$[4.5 ; 7.3]$} & $5.6 \pm 1.8$ & {$[4.5 ; 6.7]$} & $5.4 \pm 3.7$ & {$[3.2 ; 7.7]$} \\
\hline & CG & $\mathrm{G} \quad 7.4 \pm 2.4$ & {$[5.7 ; 9.0]$} & $5.7 \pm 1.8$ & {$[4.4 ; 7.0]$} & $6.9 \pm 3.8$ & {$[4.3 ; 9.6]$} \\
\hline \multirow[t]{2}{*}{ Complex DT walking } & IG & $8.2 \pm 3.8$ & {$[5.9 ; 10.5]$} & $6.6 \pm 5.4$ & {$[3.3 ; 9.9]$} & $8.3 \pm 5.3$ & {$[5.1 ; 11.6]$} \\
\hline & CG & $9.2 \pm 3.9$ & {$[6.4 ; 11.9]$} & $9.3 \pm 5.5$ & {$[5.4 ; 13.2]$} & $10.0 \pm 5.4$ & {$[6.2 ; 13.9]$} \\
\hline
\end{tabular}

improvements on the costs induced by the complex dual task were observed for gait speed and gait variabilities, with both demonstrating relative stability over a period of 3 months (although somewhat reduced).

Dual-task and gait

The current findings confirm the existing link between cognition and gait performance (e.g., $[5,8,9])$. We found significant deteriorations of walking performance under the complex DT condition for gait speed and stride time variabilities, but not for stride length variabilities (although suggestive). The present findings corroborate previous data (e.g., [24, 25]), as gait abnormalities (and hence, fall risk) increase with the complexity of the secondary task.

\section{Cognitive training and gait}

With the present study, we aimed at improving gait parameters experienced by old adults under complex DT situations. We obtained several results pointing in the expected direction. We observed small to large clinically meaningful changes in the IG after the training with improved gait parameters under complex DT situations. This suggests that participants in the IG experience a weaker negative influence of the complex cognitive task on their gait performance after the intervention. The present data corroborates previous findings [11] on gait speed under normal and walking-while-talking situations. The IG of Verghese and colleagues (composed of communitydwelling older adults) improved their gait speed under the walking-while-talking situation by $4 \mathrm{~cm} / \mathrm{s}$, representing a small but clinically meaningful change [22]. Similar changes were observed in our IG with a mean gait speed improvement of $3.6 \mathrm{~cm} / \mathrm{s}$ (see Table 3 ) and thus extending Verghese's findings to a group of old and frail nursing home residents. 3 months after the intervention, two meaningful effects remained, (a) improvements in gait speed and (b) increase in stability of stride length under the simple DT. It is unclear if the instability of the improvements in stride time and stride length (under the complex DT situation) are due to specific characteristics of the 
Fig. 2 Mean dual-task costs in gait speed (a), Stride time variability (b), and Stride length variability (c) at pre-

intervention, post-intervention, and follow-up assessments for the IG $(n=12)$ and the CG $(n=9)$. Error bars represent standard errors of mean



b

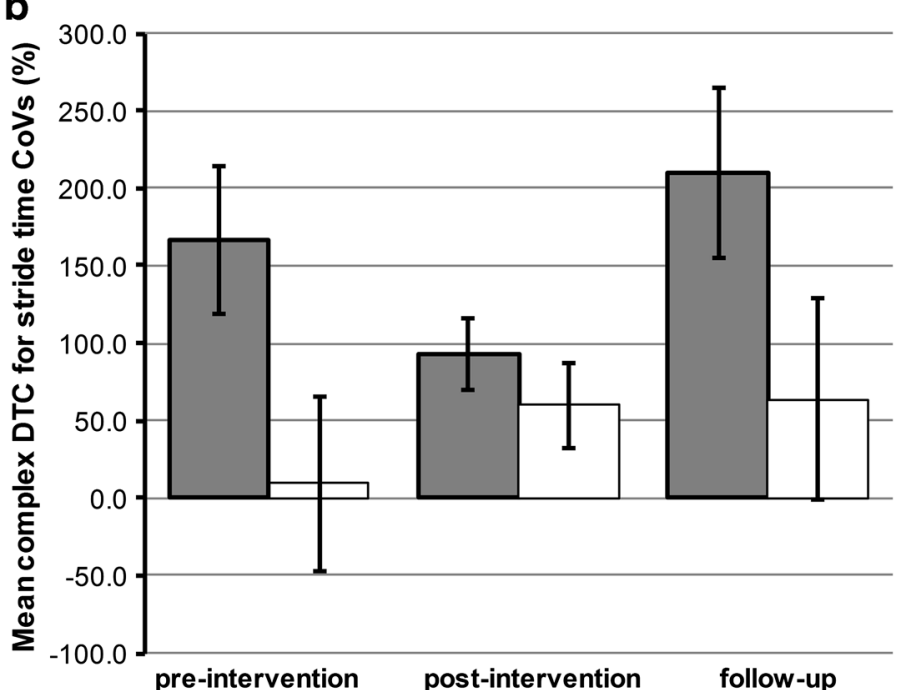

Intervention group $\square$ Control group

Intervention group

$\square$ Control group 
present sample or the cognitive training program. Research focusing on long-term training effects will need to disentangle these factors. A recent study which implemented a 12-month cognitive training program observed relatively large training gains, but they appeared only after 9 months of the program and remained constant for the following 6 months [26]. Hence, this may indicate that increasing the duration of cognitive training programs may lead not only to larger but also to more stable effects in time.

Given the pilot nature of the present study, our investigation demonstrates a number of limitations. First, the restricted sample size limits us from producing clear-cut conclusions and prohibits us from generalizing the present findings to a larger population. An anonymous reviewer suggested that we increase our sample size; while we completely agree that large samples sizes from clinical populations are highly desirable, we contend that small sample studies (as this one) in an emerging field of research are crucial for spurring future research and clinical studies. Second, some baseline gait parameters in the IG differed from parameters in the CG (CoVs). It remains unclear to what factors these baseline differences in gait parameters are attributable, especially as investigations of gait were performed for both groups according to the exact same protocol with the same investigator and gait data were double-checked for errors. Note that both groups did not significantly differ in terms of their overall characteristics (see Table 1). Although controlling a posteriori for several possible influences by means of covariates, the remaining baseline differences are likely to be attributable to our not completely randomized samples. More precisely, it can be said that the (quasi-) randomization of our samples was biased by patients who were not willing to participate in the IG, but agreed to participate as a control in the study. This shortcoming may have influenced the present data in an unforeseen and non-random manner. Hence and because of this bias, we are unable to account for all possible a priori differences between groups, i.e., unobserved heterogeneity that was not taken into account in the present study. Taken together, these limitations prohibit us from concluding definitively on the positive influence of the cognitive training program on gait, but our results are suggestive of such a claim. Thus, following the present pilot study, we have created a larger interdisciplinary research group that aims at studying more precisely the effects of structured cognitive training programs on different levels (e.g., cognitive and functional level) in older adults, using a number of different research methods.

\section{Conclusion}

The present pilot study's approach of improving gait under challenging walking situations by interventions designed to improve cognition adds encouraging results to this promising field of research. Importantly, findings of the present study go in the expected direction suggesting that cognitive intervention effects may transfer to an untrained gait domain. Our data corroborates previous findings (see gaitcognition interaction, DT complexity) and extends them to the group of frail old nursing home residents. In conclusion, the data, although preliminary, is encouraging and thus warrants further investigations.

Acknowledgments The authors are grateful to Nissrine Benabad, MSc and Véronique Cornu, MSc for their support during data collection and training sessions. The authors furthermore want to thank the residents for their participation in the study and the long-term care staff for their support. We thank Dr. Christine Schiltz and Dr. Salvador Rivas for their valuable time and for proofreading the manuscript.

Conflict of interest On behalf of all authors, the corresponding author states that there is no conflict of interest.

Open Access This article is distributed under the terms of the Creative Commons Attribution License which permits any use, distribution, and reproduction in any medium, provided the original author(s) and the source are credited.

\section{References}

1. Segev-Jacubovski O, Herman T, Yogev-Seligmann G, Mirelman A, Giladi N, Hausdorff JM (2011) The interplay between gait, falls and cognition: can cognitive therapy reduce fall risk? Expert Rev Neurother 11:1057-1075. doi:10.1586/ern.11.69

2. Rosso AL, Studenski SA, Chen WG, Aizenstein HJ, Alexander NB, Bennett DA, Black SE, Camicioli R, Carlson MC, Ferrucci L, Guralnik JM, Hausdorff JM, Kaye J, Launer LJ, Lipsitz LA, Verghese J, Rosano C (2013) Aging, the central nervous system, and mobility. J Gerontol A Biol Sci Med Sci 68(11):1379-1386. doi:10.1093/gerona/glt089

3. Herman T, Mirelman A, Giladi N, Schweiger A, Hausdorff JM (2010) Executive control deficits as a prodrome to falls in healthy older adults: a prospective study linking thinking, walking, and falling. J Gerontol A Biol Sci Med Sci 65A:1086-1092. doi:10. 1093/gerona/glq077

4. Mirelman A, Herman T, Brozgol M, Dorfman M, Sprecher E, Schweiger A, Giladi N, Hausdorff JM (2012) Executive function and falls in older adults: new findings from a five-year prospective study link fall risk to cognition. PLoS One. doi:10.1371/ journal.pone.0040297

5. Montero-Odasso $\mathrm{M}$, Verghese $\mathrm{J}$, Beauchet $\mathrm{O}$, Hausdorff JM (2012) Gait and cognition: a complementary approach to understanding brain function and the risk of falling. J Am Geriatr Soc 60:2127-2136. doi:10.1111/j.1532-5415.2012.04209.x

6. Woollacott M, Shumway-Cook A (2002) Attention and the control of posture and gait: a review of an emerging area of research. Gait Posture 16:1-14

7. Taylor ME, Ketels MM, Delbaere K, Lord SR, Mikolaizak AS, Close JCT (2012) Gait impairment and falls in cognitively impaired older adults: an explanatory model of sensorimotor and neuropsychological mediators. Age Ageing 41:665-669. doi:10. 1093/ageing/afs057

8. Hausdorff JM, Yogev G, Springer S, Simon ES, Giladi N (2005) Walking is more like catching than tapping: gait in the elderly as 
a complex cognitive task. Exp Brain Res 164:541-548. doi:10. 1007/s00221-005-2280-3

9. Yogev-Seligmann G, Hausdorff JM, Giladi N (2008) The role of executive function and attention in gait. Mov Disord 23:329-342. doi: $10.1002 / \mathrm{mds} .21720$

10. Pichierri G, Wolf P, Murer K, de Bruin ED (2011) Cognitive and cognitive-motor interventions affecting physical functioning: a systematic review. BMC Geriatr 11:29. doi:10.1186/1471-231811-29

11. Verghese J, Mahoney J, Ambrose AF, Wang C, Holtzer R (2010) Effect of cognitive remediation on gait in sedentary seniors. J Gerontol A Biol Sci Med Sci 65A:1338-1343. doi:10.1093/ gerona/glq127

12. Rebok GW, Ball K, Guey LT, Jones RN, Kim H-Y, King JW, Marsiske M, Morris JN, Tennstedt SL, Unverzagt FW, Willis SL (2014) Ten-year effects of the advanced cognitive training for independent and vital elderly cognitive training trial on cognition and everyday functioning in older adults. J Am Geriatr Soc. doi:10.1111/jgs.12607

13. Baller G, Kalbe E, Kaesberg S, Kessler J (2009) NEUROvitalis. Neuropsychologisches Gruppentraining. (NEUROvitalis. Neuropsychological group training). Prolog, Cologne, Germany

14. Kressig RW, Beauchet O, European GAITRite Network Group (2006) Guidelines for clinical applications of spatio-temporal gait analysis in older adults. Aging Clin Exp Res 18:174-176

15. Schwenk M, Schmidt M, Pfisterer M, Oster P, Hauer K (2011) Rollator use adversely impacts on assessment of gait and mobility during geriatric rehabilitation. J Rehabil Med 43:424-429. doi:10.2340/16501977-0791

16. Hausdorff JM (2007) Gait dynamics, fractals and falls: finding meaning in the stride-to-stride fluctuations of human walking. Hum Mov Sci 26:555-589. doi:10.1016/j.humov.2007.05.003

17. D'Ath P, Katona P, Mullan E, Evans S, Katona C (1994) Screening, detection and management of depression in elderly primary care attenders. I: The acceptability and performance of the 15 item Geriatric Depression Scale (GDS15) and the development of short versions. Fam Pract 11:260-266
18. Miller MD, Paradis CF, Houck PR, Mazumdar S, Stack JA, Rifai AH, Mulsant B, Reynolds CF (1992) Rating chronic medical illness burden in geropsychiatric practice and research: application of the cumulative illness rating scale. Psychiatry Res 41:237-248

19. Kalbe E, Kaesberg S, Mayer A, Schlegel M, Baller G, Fink G, Kessler J (2009) NeuroVitalis ${ }^{\circledR}$ : effekte eines neuen neuropsychologischen Trainings bei Patienten mit leichten kognitiven Störungen und Patienten mit Alzheimer Demenz [NeuroVitalis ${ }^{\circledR}$ : Effects of a new neuropsychological training in patients with mild cognitive impairments and patients with Alzeihmer's disease]. Aktuelle Neurol. doi:10.1055/s-0029-1238381

20. Hoaglin DC, Iglewicz B, Tukey JW (1986) Performance of some resistant rules for outlier labeling. J Am Stat Assoc 81:991-999. doi: $10.2307 / 2289073$

21. Kazis LE, Anderson JJ, Meenan RF (1989) Effect sizes for interpreting changes in health status. Med Care 27:178-189

22. Perera S, Mody SH, Woodman RC, Studenski SA (2006) Meaningful change and responsiveness in common physical performance measures in older adults. $\mathrm{J}$ Am Geriatr Soc 54:743-749. doi:10.1111/j.1532-5415.2006.00701.x

23. Cohen J (1988) Statistical power analysis for the behavioral sciences. L. Erlbaum Associates, Hillsdale, NJ, USA

24. Montero-Odasso M, Muir SW, Speechley M (2012) Dual-task complexity affects gait in people with mild cognitive impairment: the interplay between gait variability, dual tasking, and risk of falls. Arch Phys Med Rehabil 93:293-299. doi:10.1016/j.apmr. 2011.08.026

25. Beauchet O, Dubost V, Aminian K, Gonthier R, Kressig RW (2005) Dual-task-related gait changes in the elderly: does the type of cognitive task matter? J Mot Behav 37:259-264

26. Salotti P, Sanctis BD, Clementi A, Ferreira MF, Silvestris TD (2013) Evaluation of the efficacy of a cognitive rehabilitation treatment on a group of Alzheimer's patients with moderate cognitive impairment: a pilot study. Aging Clin Exp Res 25:403-409. doi:10.1007/s40520-013-0062-5 